\title{
A national FFQ for the Netherlands (the FFQ-NL 1.0): validation of a comprehensive FFQ for adults
}

\author{
Diewertje Sluik $^{1 *}$, Anouk Geelen ${ }^{1}$, Jeanne H. M. de Vries ${ }^{1}$, Simone J. P. M. Eussen ${ }^{2}$, Henny A. M. Brants ${ }^{3}$, \\ Saskia Meijboom ${ }^{1}$, Martien C. J. M. van Dongen ${ }^{2}$, H. Bas Bueno-de-Mesquita ${ }^{3,4,5,6}$, \\ Nicole E. G. Wijckmans-Duysens ${ }^{2}$, Pieter van 't Veer', Pieter C. Dagnelie ${ }^{2} \dagger$, Marga C. Ocké ${ }^{3} \dagger$ \\ and Edith J. M. Feskens ${ }^{1} \dagger$ \\ ${ }^{1}$ Division of Human Nutrition, Wageningen University, PO Box 17, 6700 AA Wageningen, The Netherlands \\ ${ }^{2}$ Department of Epidemiology, Care and Public Health Research Institute/Cardiovascular Research Institute (CAPHRI/CARIM), \\ Maastricht University, PO Box 616, 6200 MD Maastricht, The Netherlands \\ ${ }^{3}$ National Institute for Public Health and the Environment, PO Box 1, 3720 BA Bilthoven, The Netherlands \\ ${ }^{4}$ Department of Gastroenterology and Hepatology, University Medical Centre, PO Box 85500, 3508 GA Utrecht, The Netherlands \\ ${ }^{5}$ Department of Epidemiology and Biostatistics, The School of Public Health, Imperial College London, South Kensington \\ Campus, London SW7 2AZ, UK \\ ${ }^{6}$ Department of Social and Preventive Medicine, Faculty of Medicine, University of Malaya, 50603 Kuala Lumpur, Malaysia
}

(Submitted 2 February 2016 - Final revision received 11 May 2016 - Accepted 23 June 2016 - First published online 25 July 2016)

\section{Abstract}

A standardised, national, 160-item FFQ, the FFQ-NL 1.0, was recently developed for Dutch epidemiological studies. The objective was to validate the FFQ-NL 1.0 against multiple 24-h recalls (24hR) and recovery and concentration biomarkers. The FFQ-NL 1.0 was filled out by 383 participants (25-69 years) from the Nutrition Questionnaires plus study. For each participant, one to two urinary and blood samples and one to five (mean 2.7) telephone-based $24 \mathrm{hR}$ were available. Group-level bias, correlation coefficients, attenuation factors, de-attenuated correlation coefficients and ranking agreement were assessed. Compared with the $24 \mathrm{hR}$, the FFQ-NL 1.0 estimated the intake of energy and macronutrients well. However, it underestimated intakes of SFA and trans-fatty acids and alcohol and overestimated intakes of most vitamins by $>5 \%$. The median correlation coefficient was 0.39 for energy and macronutrients, 0.30 for micronutrients and 0.30 for food groups. The FFQ underestimated protein intake by an average of $16 \%$ and $\mathrm{K}$ by $5 \%$, relative to their urinary recovery biomarkers. Attenuation factors were 0.44 and 0.46 for protein and $\mathrm{K}$, respectively. Correlation coefficients were $0.43-0.47$ between (fatty) fish intake and plasma EPA and DHA and 0.24-0.43 between fruit and vegetable intakes and plasma carotenoids. In conclusion, the overall validity of the newly developed FFQ-NL 1.0 was acceptable to good. The FFQ-NL 1.0 is well suited for future use within Dutch cohort studies among adults.

Key words: 24-h recall: Concentration biomarkers: FFQ: Measurement errors: Recovery biomarkers: Validation studies

In nutritional epidemiology, prospective cohort studies constitute the strongest observational design to study associations between diet and health outcomes ${ }^{(1)}$. FFQ are the common choice for assessing dietary intake in large observational studies: they are able to capture usual, individual, long-term dietary intake, and participant burden is low ${ }^{(2)}$. However, FFQ rely on long-term memory and are subject to socially desirable answers ${ }^{(1)}$. Moreover, certain foods may be neglected because of the fixed food list ${ }^{(1)}$. As a result of these types of measurement errors, FFQ may not be useful to detect weak associations between dietary intake and health outcomes ${ }^{(3,4)}$.
In order to stimulate standardised assessment of dietary intake in large-scale studies in the Netherlands, a new FFQ has been developed - the FFQ-NL 1.0. This FFQ aims to provide comprehensive and standardised data collection of usual energy, food and nutrient intakes in Dutch adults. The questionnaire has been developed to suit current and future research objectives for epidemiological research in the Netherlands. Moreover, to incorporate changes in dietary habits and food products over time, the most recent Dutch National Food Consumption Survey and food composition table were used. The development of the FFQ-NL 1.0 and its compatibility with other Dutch FFQ will be

Abbreviations: 24hR, 24-h recall; EPIC, European Prospective Investigation into Cancer and Nutrition; ICC, intraclass correlation coefficient; PABA, paraaminobenzoic acid.

* Corresponding author: D. Sluik, fax +31 3174 82782, email Diewertje.Sluik@wur.nl

$\dagger$ These authors contributed equally to this work. 
described separately (SJPM Eussen, MCJM van Dongen, NEG Wijckmans-Duysens, et al., unpublished results). The FFQ-NL 1.0 will become nationally available for use in future Dutch epidemiological studies and as such it may serve as a national reference FFQ. Validation of this newly developed FFQ is essential to provide insight to which extent the measurement errors distort observed diet-disease relationships ${ }^{(5)}$. The objective of this study was to validate the FFQ-NL 1.0 in adults against multiple 24-h dietary recalls (24hR), 24-h $\mathrm{N}$ and $\mathrm{K}$ excretion in urine, and plasma carotenoids and fatty acids measured in cholesteryl esters.

\section{Methods \\ Study design and population}

The validation study was embedded in the Nutrition Questionnaires plus (NQplus) study, an ongoing longitudinal study in the city of Wageningen and surroundings, the Netherlands ${ }^{(6)}$. Between 2011 and 2013, 2048 men and women aged 20-70 years were included. The NQplus study was approved by the ethics committee of Wageningen University and conducted according to the guidelines laid down in the Declaration of Helsinki. Participants filled out general and health questionnaires at baseline, year 1 and year 2. Moreover, a physical examination including blood and urine collection was performed at baseline, year 1 and year 2, and multiple 24hR were administered throughout the 2-year study period. All participants provided their written informed consent. For the present validation study, a sample of 445 participants aged 25-69 years was invited to fill out the FFQ-NL 1.0, of which 386 (87\%) responded. A random subsample of 150 people agreed to repeat urine and blood tests. Fig. 1 gives an overview of the measurements and the time frame for the purpose of this validation study.

\section{FFQ-NL 1.0}

The National FFQ for the Netherlands, the FFQ-NL 1.0, was developed to obtain comprehensive and standardised data on food intake in large samples of Dutch adults. The FFQ-NL 1.0 was developed with FFQTOOL ${ }^{\mathrm{TM}}$, an online tool to develop tailor-made FFQ, and was designed for use in the general population. The food items in the FFQ covered $\geq 85 \%$ of the absolute intake of energy and thirty-nine nutrients, $\geq 88 \%$ of the inter-individual variation in intake of energy and macronutrients and $45-93 \%$ of the inter-individual variation in intake of micronutrients as assessed by two non-consecutive $24 \mathrm{hR}$ in the Dutch National Food Consumption Survey 2007-2010 ${ }^{(7)}$. The FFQ-NL 1.0 consists of 160 food items with questions on frequency and consumed amounts with a 1-year reference period. Average daily energy and nutrient intakes were calculated by multiplying frequency of consumption by consumed amounts and nutrient content per item using the Dutch food composition table of $2011^{(8)}$. Of the 386 participants, three were excluded on the basis of their energy intakes: $<2092 \mathrm{~kJ} / \mathrm{d}(<500 \mathrm{kcal} / \mathrm{d})$ or $>14644 \mathrm{~kJ} / \mathrm{d}(>3500 \mathrm{kcal} / \mathrm{d})$ in women and $<3347 \mathrm{~kJ} / \mathrm{d}$ $(<800 \mathrm{kcal} / \mathrm{d})$ or $>16736 \mathrm{~kJ} / \mathrm{d}(>4000 \mathrm{kcal} / \mathrm{d})$ in $\operatorname{men}^{(9)}$. A total of 278 persons filled out the FFQ again after about 12 months.

\section{4-h recalls}

As part of the overall NQplus study, multiple, telephone-based $24 \mathrm{hR}$ were administered. Dates were randomly selected and scheduled evenly across the year and days of the week. The $24 \mathrm{hR}$ were administered by dietitians trained in interviewing skills using a five-step, multiple-pass method, which is a validated technique to increase accuracy ${ }^{(10-12)}$. Recalls were transcribed into food codes and food groups of the 2011 Dutch food composition table ${ }^{(13)}$. For each person, individually, recalls that were assessed within 12 months before that person filled out the FFQ-NL 1.0 for the first time were selected, resulting in 1038 $24 \mathrm{hR}$. For seven persons, no $24 \mathrm{hR}$ were available, whereas for the remaining 376 persons, an average of $2 \cdot 7$ (range $1-5$ ) $24 \mathrm{hR}$ was included in the current analyses.

\section{Urine collection and analyses}

The 24-h urine collections started with the second voiding after waking up and were completed with the first voiding after waking up the next day. Urine samples were handed in at the hospital and transported to the study centre, where they were mixed, weighed, aliquoted and stored at $-20^{\circ} \mathrm{C}$ until further analysis. The participants received three 80-mg para-aminobenzoic acid (PABA) tablets to check for completeness of the urine collections. PABA recovery was measured using the HPLC method $^{(14)}$. A recovery of at least $78 \%$ of the ingested PABA was considered as complete urine collection. Within the Observing Protein and Energy Nutrition Study, the exclusion of participants with incomplete urine samples had little or no effect on the correlation with true intake and the attenuation factors derived for the $\mathrm{FFQ}^{(15)}$. Hence, the analyses were performed on all urine samples. In a sensitivity analysis, persons with a PABA recovery $<78 \%$ ( $n$ 82) were excluded. Total 24-h N excretion was determined by the Kjeldahl technique (Foss Kjeltec ${ }^{\mathrm{TM}} 2300$ analyser; Foss Analytical). Urinary protein content was calculated using the following formula:

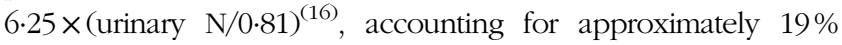
faecal and skin losses. Urinary $\mathrm{K}$ concentration measurements were performed with an ion-selective electrode on a Roche 917 analyser; urinary excretion of $81 \%$ was assumed ${ }^{(17)}$. Urinary $\mathrm{N}$ and $\mathrm{K}$ were available for 363 persons, of which 139 provided a replicate urine sample to determine urinary $\mathrm{N}$ and $\mathrm{K}$.

\section{Blood collection and analyses}

After a 10-h overnight fast, $24 \mathrm{ml}$ of blood was drawn from an antecubital vein using venepuncture. Blood was immediately centrifuged, and plasma was stored at $-80^{\circ} \mathrm{C}$ until further analyses. Carotenoids were determined using HPLC and UV-vis detection $^{(18)}$. Fatty acids from plasma cholesteryl esters were quantified by GLC using the solid-phase extraction method to separate the cholesteryl esters with acidified methanol. Peak retention times and area percentages of total fatty acids were determined by using known cholesteryl ester standards and analysed using Agilent Technologies ChemStation software (Agilent) ${ }^{(19)}$. Plasma carotenoids were available for 360 persons and plasma fatty acids for 358 persons, of which fatty acids and carotenoids were determined in replicate blood samples of 141 persons. 


\section{Other variables}

Height was measured without shoes using a stadiometer (SECA 213; SECA Corp.). Weight was measured without shoes and heavy clothing and with empty pockets on a digital scale (SECA 877; SECA Corp.). Questionnaires assessed educational level, presence of diseases, smoking status and whether the participants followed a diet regimen.

\section{Measurement error models}

It was assumed that dietary intake estimated using multiple $24 \mathrm{hR}$ as well as protein and $\mathrm{K}$ intakes estimated by urinary analysis were the best standards to approximate true intake ${ }^{(20)}$. For the replicate FFQ-NL 1.0, a constant bias, intake-related bias and person-specific bias were assumed to be present. The following measurement error models were used:

$$
\begin{array}{ll}
\text { 24-h recall }(R): & R=T+e(R) \\
\text { Urinary biomarker }(G): & G=T+e(G)
\end{array}
$$$$
\text { FFQ-NL } 1.0(Q): \quad Q=A(Q)+B(Q) \times T+q+e(Q),
$$

where $A$ is the constant bias, $B$ the intake-related bias; $e$ the random error, $q$ the person-specific bias, FFQ-NL 1.0 and $T$ the true (unknown) intake.

\section{Statistical analyses}

All statistical analyses were performed using SAS 9.3. Linear mixed models with a random intercept for participants were applied taking into account multiple measurements per person. Macronutrients and alcohol were additionally expressed in energy densities to adjust for energy. Absolute differences between self-reported intakes in the FFQ-NL 1.0 and intakes estimated from the reference methods - that is $24 \mathrm{hR}$, urinary biomarker or replicate FFQ - were expressed as group-level bias: (mean intake FFQ-NL 1.0/mean intake reference method) $\times 100-100$; differences larger than 5\% were considered relevant. For protein and $\mathrm{K}$, bias in mean intake was evaluated by comparing the distributions of reported intake and intake based on urinary excretion. The attenuation factor was estimated as the slope in the linear regression of the reference method on the reported intake according to the FFQ-NL 1.0. The validity coefficient or de-attenuated correlation coefficient is defined as the correlation between the observed intake as measured using the FFQ and the 'true' intake as measured using the biomarkers and $24 \mathrm{hR}$. The de-attenuated correlation coefficient was estimated as the correlation coefficient between the FFQ-NL 1.0 and the reference instrument divided by the square root of the intraclass correlation coefficient (ICC) of replicates of the reference method $^{(21,22)}$. The validity of the FFQ-NL 1.0 was judged based on comparison with other published FFQ and whether the correlation coefficients fell within the range of $0.4-0.7$ as mentioned by Willett ${ }^{(23)}$. Validity was first evaluated by attenuation factors and de-attenuated correlation coefficients for protein and $\mathrm{K}$ using recovery biomarkers and for other nutrients and foods using $24 \mathrm{hR}$ as the reference method, followed by reproducibility and ranking ability.
The replicate FFQ-NL 1.0 was used to study reproducibility only. In addition to the unstratified analysis, stratified analyses were performed for men and women, persons aged 25-56 and 57-69 years (median split), according to educational status (low/middle: no, lower or lower vocational education; intermediate: intermediate vocational; and high: higher vocational or university), and for persons with $\mathrm{BMI}<25$ and $\geq 25 \mathrm{~kg} / \mathrm{m}^{2}$.

\section{Results}

\section{Timing of the measurements and general characteristics}

The FFQ-NL 1.0 was administered throughout the year, with a minor higher frequency during winter (Fig. 1). The FFQ-NL 1.0 was repeated after approximately 1 year. Blood and urine sample collections and $24 \mathrm{hR}$ were spread out equally over the seasons. The mean age of the participants was 53.9 (SD 10.4) years and $61 \%$ were women (Table 1 ). In total, $45 \%$ of the subjects were overweight or obese. Most of the participants were highly educated (60\%), and a few participants had prevalent diseases (13\%).

\section{Validation of nutrients using 24-h recall}

Compared with the $24 \mathrm{hR}$, group-level bias was small for energy, most macronutrients, water and dietary fibre (Table 2). The FFQ-NL 1.0 underestimated the intake of total fat (g), SFA, trans-fatty acids and $\mathrm{Ca}$ and overestimated the intake of alcohol, EPA, DHA, haem-iron and most vitamins. For energy, macronutrients, dietary fibre and water, de-attenuated correlation coefficients ranged from 0.26 for trans-fatty acids to $1 \cdot 18$ for dietary fibre. For micronutrients, correlations ranged from 0.38 for vitamin $\mathrm{B}_{1}$ to 0.65 for $\mathrm{Mg}$. For energy, macronutrients, dietary fibre and water, attenuation factors ranged from 0.32 for MUFA to 0.73 for alcohol and for micronutrients from 0.27 for vitamin $\mathrm{B}_{1}$, folic acid and haem-iron to 0.48 for $\mathrm{Mg}$. Group-level bias of nutrients was higher in older than in younger subjects (data not shown). Attenuation factors were similar between age categories and men and women, but higher in highly educated subjects and subjects with a normal BMI (online Supplementary Table S1). De-attenuated correlations were higher in men, in highly educated subjects and in subjects with overweight and obesity (online Supplementary Table S2).

\section{Validation of food groups using 24- $h$ recall}

For food groups, group-level bias was small (defined as $<5 \%$ ) for the intakes of non-alcoholic beverages, bread, fruit, nuts/seeds/snacks, soup and fats/oils/sauces (Table 3). The FFQ-NL 1.0 underestimated the intakes of cake/cookies, vegetables, cheese, composite dishes and sugar/honey/jams/candy and overestimated the intakes of potatoes, eggs, cereals, savoury sandwich fillings, milk and milk products, legumes, soya and vegetarian products, fish and meat. Attenuation factors varied between 0.13 (legumes) and 0.72 (soya and vegetarian products). 


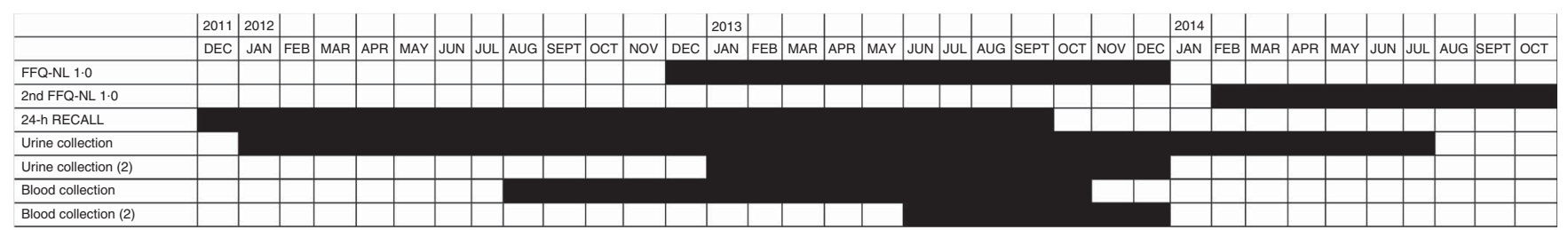

Fig. 1. Time frame and overview of measurements of the FFQ-NL 1.0 validation study.

Table 1. General characteristics of the 383 NQplus participants, aged 25-69 years, included in the FFQ-NL 1.0 validation study (Mean values and standard deviations; numbers and percentages)

\begin{tabular}{|c|c|c|c|c|c|c|}
\hline & \multicolumn{2}{|c|}{ All } & \multicolumn{2}{|c|}{ Men } & \multicolumn{2}{|c|}{ Women } \\
\hline & Mean & SD & Mean & SD & Mean & SD \\
\hline$n$ & \multicolumn{2}{|c|}{383} & \multicolumn{2}{|c|}{149} & \multicolumn{2}{|c|}{234} \\
\hline Age (years) & 53.9 & $10 \cdot 4$ & $56 \cdot 9$ & $9 \cdot 2$ & $52 \cdot 0$ & $10 \cdot 7$ \\
\hline $\mathrm{BMI}\left(\mathrm{kg} / \mathrm{m}^{2}\right)$ & $25 \cdot 4$ & $4 \cdot 1$ & $26 \cdot 0$ & $3 \cdot 3$ & 24.9 & 4.5 \\
\hline Waist (cm) & $89 \cdot 6$ & $12 \cdot 3$ & $95 \cdot 5$ & $10 \cdot 4$ & $85 \cdot 7$ & $11 \cdot 8$ \\
\hline \multirow[t]{2}{*}{ Waist:hip ratio } & 0.87 & 0.09 & 0.93 & 0.07 & 0.83 & 0.07 \\
\hline & $n$ & $\%$ & $n$ & $\%$ & $n$ & $\%$ \\
\hline \multicolumn{7}{|l|}{ BMI } \\
\hline Normal weight $\left(<25 \mathrm{~kg} / \mathrm{m}^{2}\right)$ & 208 & 54 & 65 & 44 & 143 & 61 \\
\hline Overweight $\left(25-29.9 \mathrm{~kg} / \mathrm{m}^{2}\right)$ & 131 & 34 & 65 & 44 & 66 & 28 \\
\hline Obese $\left(\geq 30 \mathrm{~kg} / \mathrm{m}^{2}\right)$ & 44 & 11 & 19 & 13 & 25 & 11 \\
\hline \multicolumn{7}{|l|}{ Educational attainment } \\
\hline Low & 14 & 4 & 6 & 4 & 8 & 3 \\
\hline Medium & 137 & 36 & 45 & 30 & 92 & 40 \\
\hline High & 230 & 60 & 98 & 66 & 132 & 57 \\
\hline \multicolumn{7}{|l|}{ Smoking status } \\
\hline Former & 171 & 51 & 55 & 42 & 116 & 56 \\
\hline Never & 146 & 43 & 63 & 48 & 83 & 40 \\
\hline Current & 21 & 6 & 12 & 9 & 9 & 4 \\
\hline \multicolumn{7}{|l|}{ Disease history } \\
\hline None & 334 & 87 & 125 & 86 & 209 & 89 \\
\hline Myocardial Infarction & 7 & 2 & 5 & 3 & 2 & 1 \\
\hline Stroke & 6 & 2 & 6 & 4 & 0 & 0 \\
\hline Diabetes & 13 & 3 & 7 & 5 & 6 & 3 \\
\hline Cancer & 26 & 7 & 8 & 5 & 18 & 8 \\
\hline \multicolumn{7}{|l|}{ Diet regimen } \\
\hline Yes, always & 13 & 3 & 5 & 4 & 8 & 3 \\
\hline Sometimes & 13 & 3 & 5 & 4 & 8 & 3 \\
\hline No & 348 & 93 & 133 & 93 & 215 & 92 \\
\hline
\end{tabular}

\section{Validation of protein and potassium using urinary}

\section{biomarkers}

Compared with the urinary recovery biomarkers, the FFQ-NL 1.0 underestimated protein intake by $15.9 \%$ and $\mathrm{K}$ intake by $4.8 \%$ at the group level (Table 4); this is confirmed in Fig. 2 and 3. The attenuation factors for protein and $\mathrm{K}$ were 0.46 (95\% CI $0.35,0.57)$ and 0.44 (95\% CI $0.32,0.55)$, respectively. De-attenuated correlation coefficients were 0.69 (95\% CI 0.53 , 0.83 ) for protein and 0.58 (95\% CI $0.43,0.73)$ for $\mathrm{K}$. Including only subjects with a PABA recovery $\geq 78 \%$ ( $n$ 280) yielded lower validity measures: attenuation factors were 0.42 (95\% CI 0.29 , $0.55)$ for protein and 0.39 (95\% CI $0.25,0.53)$ for $\mathrm{K}$, and de-attenuated correlation coefficients were 0.59 (95\% CI 0.42 , $0.74)$ for protein and 0.47 (95\% CI $0.31,0.63)$ for K. Attenuation factors for protein and $\mathrm{K}$ tended to be higher among younger subjects, in men and in subjects with higher education and normal BMI (online Supplementary Tables S1 and S2).
The underestimation of protein and $\mathrm{K}$ intakes at a group level was larger in younger persons and slightly higher among subjects with higher BMI and higher education. Group-level bias for protein was higher in men and for $\mathrm{K}$ higher among women (data not shown).

\section{Validation of (fatty) fish, fruit and vegetable intakes using concentration biomarkers}

Correlation coefficients between (fatty) fish intake and plasma $n-3$ fatty acids were $0 \cdot 43-0 \cdot 47$ (Table 5 ). Correlation coefficients between plasma carotenoids and fruit and vegetable intakes ranged between 0.24 and 0.43 (Table 5). Cross-classification showed that more than $60 \%$ of the participants were allocated to the same or adjacent quintile of intake or plasma concentration. The correlations between fruit and vegetable intake and total carotenoids were somewhat higher among younger subjects and women. For fish intake and plasma 
Table 2. Absolute intakes of nutrients in the FFQ-NL 1.0 and telephone-based 24 -h recalls $(24 \mathrm{hR})$ and the relative difference, correlation coefficients, attenuation factors, de-attenuated correlation coefficients and cross-classifiction between the FFQ-NL 1.0 and the telephone-based 24 -h recalls*

(Mean values with their standard errors; group-level bias, correlation coefficient, attenuation factor, de-attenuated correlation coefficient and $95 \%$ confidence intervals)

\begin{tabular}{|c|c|c|c|c|c|c|c|c|c|c|c|c|c|c|}
\hline & \multicolumn{4}{|c|}{ Absolute intake } & \multirow[b]{3}{*}{$\begin{array}{c}\text { Group-level } \\
\text { bias (\%) }\end{array}$} & \multirow[b]{3}{*}{$95 \% \mathrm{Cl}$} & \multirow[b]{3}{*}{$\begin{array}{l}\text { Correlation } \\
\text { coefficient }\end{array}$} & \multirow[b]{3}{*}{$95 \% \mathrm{Cl}$} & \multirow[b]{3}{*}{$\begin{array}{l}\text { Attenuation } \\
\text { factor }\end{array}$} & \multirow[b]{3}{*}{$95 \% \mathrm{Cl}$} & \multirow[b]{3}{*}{$\begin{array}{l}\text { De-attenuated } \\
\text { correlation coefficient }\end{array}$} & \multirow[b]{3}{*}{$95 \% \mathrm{Cl}$} & \multirow{2}{*}{\multicolumn{2}{|c|}{ Cross-classification }} \\
\hline & \multicolumn{2}{|c|}{ FFQ-NL 1.0} & \multicolumn{2}{|c|}{$24 \mathrm{hR}$} & & & & & & & & & & \\
\hline & Mean & $\mathrm{SE}$ & Mean & $\mathrm{SE}$ & & & & & & & & & $\begin{array}{c}\text { Same or } \\
\text { adjacent } Q(\%)\end{array}$ & $\begin{array}{c}\text { Extreme } \\
Q(\%)\end{array}$ \\
\hline$n$ & \multicolumn{2}{|c|}{383} & \multicolumn{2}{|c|}{376} & \multicolumn{2}{|c|}{336} & \multicolumn{2}{|c|}{376} & \multicolumn{2}{|c|}{376} & \multicolumn{2}{|l|}{376} & 376 & 376 \\
\hline Energy (kJ) & 840 & 490 & 8657 & 96 & $-9 \cdot 2$ & $-15 \cdot 4,-2 \cdot 5$ & 1.80 & $1 \cdot 26,2 \cdot 14$ & 1.67 & $1 \cdot 38,2 \cdot 01$ & $2 \cdot 30$ & $1 \cdot 12,2 \cdot 51$ & 280 & 13 \\
\hline Energy (kcal) & 2025 & 28 & 2069 & 23 & $-2 \cdot 2$ & $-3 \cdot 7,-0.6$ & 0.43 & $0.36,0.51$ & 0.40 & $0.33,0.48$ & 0.55 & $0.44,0.65$ & 67 & 3 \\
\hline Protein (en\%) & $16 \cdot 2$ & 0.1 & $16 \cdot 1$ & 0.2 & 0.7 & $0.6,0.8$ & 0.44 & $0.37,0.51$ & 0.62 & $0.52,0.71$ & 0.59 & $0.48,0.70$ & 67 & 2 \\
\hline Protein (g) & & & & & & & & & & & & & & \\
\hline Total & 82 & 1 & 81 & 1 & $1 \cdot 8$ & $1 \cdot 4,2 \cdot 1$ & 0.38 & $0.31,0.46$ & 0.33 & $0.27,0.40$ & 0.54 & $0.41,0.66$ & 68 & 3 \\
\hline Vegetable & 34 & 1 & 34 & 1 & 1.6 & $1.3,1.8$ & 0.57 & $0.49,0.64$ & 0.55 & $0.47,0.62$ & 0.72 & $0.63,0.80$ & 72 & 2 \\
\hline Animal & 48 & 1 & 47 & 1 & $2 \cdot 0$ & $1 \cdot 6,2 \cdot 4$ & 0.40 & $0.33,0.47$ & 0.38 & $0.32,0.45$ & 0.56 & $0.44,0.67$ & 68 & 2 \\
\hline Fat (en\%) & $32 \cdot 7$ & 0.3 & $33 \cdot 3$ & 0.3 & $-1 \cdot 7$ & $-1 \cdot 8,-1 \cdot 5$ & 0.27 & $0.20,0.35$ & 0.37 & $0.27,0.46$ & 0.37 & $0.24,0.50$ & 60 & 5 \\
\hline Fat $(g)$ & & & & & & & & & & & & & & \\
\hline Total & 74 & 1 & 79 & 1 & $-6 \cdot 2$ & $-6 \cdot 6,-5 \cdot 9$ & 0.30 & $0.23,0.37$ & 0.35 & $0.27,0.44$ & 0.44 & $0.30,0.57$ & 61 & 4 \\
\hline SFA & 26 & 0 & 29 & 0 & $-13 \cdot 1$ & $-13 \cdot 4,-12 \cdot 9$ & 0.29 & $0.22,0.36$ & 0.40 & $0.30,0.50$ & 0.40 & $0.27,0.53$ & 63 & 4 \\
\hline MUFA & 26 & 1 & 27 & 0 & -1.8 & $-2 \cdot 0,-1 \cdot 5$ & 0.25 & $0.18,0.32$ & 0.32 & $0.23,0.40$ & 0.36 & $0.22,0.49$ & 58 & 5 \\
\hline PUFA & 16 & 0 & 16 & 0 & 0.8 & $0.6,1.0$ & 0.34 & $0.27,0.42$ & 0.41 & $0.32,0.49$ & 0.48 & $0.35,0.60$ & 64 & 4 \\
\hline ALA & 2 & 0 & 2 & 0 & $2 \cdot 4$ & $2 \cdot 3,2 \cdot 4$ & 0.26 & $0.19,0.33$ & 0.34 & $0.24,0.44$ & 0.37 & $0.23,0.50$ & 64 & 5 \\
\hline Linoleic acid & 13 & 0 & 13 & 0 & $1 \cdot 3$ & $1.1,1.5$ & 0.34 & $0.26,0.41$ & 0.40 & $0.31,0.48$ & 0.48 & $0.35,0.60$ & 58 & 6 \\
\hline EPA & 0.13 & 0.01 & 0.11 & 0.01 & $13 \cdot 8$ & $13 \cdot 8,13 \cdot 9$ & 0.33 & $0.27,0.39$ & 0.67 & $0.54,0.79$ & 0.60 & $0.43,0.76$ & 58 & 6 \\
\hline $\mathrm{DHA}$ & 0.19 & 0.01 & 0.16 & 0.02 & $19 \cdot 1$ & $19 \cdot 0,19 \cdot 2$ & 0.28 & $0.22,0.34$ & 0.60 & $0.47,0.73$ & 0.53 & $0.35,0.70$ & 64 & 1 \\
\hline Trans-fatty acids & 1.1 & 0.0 & 1.3 & 0.0 & -18.5 & $-18 \cdot 6,-18 \cdot 4$ & $0 \cdot 16$ & $0.09,0.22$ & 0.39 & $0.22,0.56$ & 0.26 & $0.10,0.42$ & 61 & 5 \\
\hline Cholesterol (mg) & 208 & 5 & 204 & 5 & $2 \cdot 2$ & $1 \cdot 2,3 \cdot 2$ & 0.27 & $0.21,0.34$ & 0.42 & $0.33,0.52$ & 0.46 & $0.30,0.62$ & 61 & 4 \\
\hline Carbohydrates (en\%) & $46 \cdot 7$ & 0.3 & 44.0 & 0.3 & -0.7 & $-0.8,-0.6$ & 0.51 & $0.44,0.59$ & 0.59 & $0.50,0.67$ & 0.65 & $0.55,0.74$ & 67 & 3 \\
\hline Carbohydrates (g) & & & & & & & & & & & & & & \\
\hline Total & 220 & 3 & 224 & 3 & -1.5 & $-2 \cdot 1,-0.9$ & 0.54 & $0.46,0.62$ & 0.51 & $0.44,0.59$ & 0.72 & $0.62,0.81$ & 71 & 2 \\
\hline Polysaccharides & 122 & 2 & 122 & 2 & 0.2 & $-0.3,0.7$ & 0.59 & $0.52,0.67$ & 0.53 & $0.46,0.60$ & 0.76 & $0.67,0.84$ & 73 & 2 \\
\hline $\begin{array}{l}\text { Mono/ } \\
\text { disaccharides }\end{array}$ & 98 & 2 & 101 & 2 & -3.5 & $-4 \cdot 0,-3 \cdot 1$ & 0.44 & $0.37,0.52$ & 0.50 & $0.41,0.59$ & 0.58 & $0.47,0.69$ & 66 & 3 \\
\hline Dietary fibre (g) & 24 & 0 & 24 & 0 & $2 \cdot 5$ & $2 \cdot 3,2 \cdot 7$ & 0.92 & $0.90,0.93$ & 0.56 & $0.48,0.63$ & $1 \cdot 18$ & $1 \cdot 16,1 \cdot 20$ & 72 & 2 \\
\hline Water $(\mathrm{g})$ & 2662 & 35 & 2653 & 33 & 0.6 & $-1 \cdot 3,2 \cdot 4$ & 0.50 & $0.42,0.58$ & 0.49 & $0.41,0.56$ & 0.61 & $0.51,0.69$ & 70 & 3 \\
\hline Alcohol (en\%) & 4.5 & 0.2 & 3.9 & 0.2 & 15.8 & $15 \cdot 5,16 \cdot 2$ & 0.83 & $0.75,0.90$ & 0.72 & $0.65,0.78$ & 1.01 & $0.97,1.04$ & 83 & 0 \\
\hline Alcohol (g) & $13 \cdot 0$ & 0.7 & $12 \cdot 1$ & 0.7 & 7.9 & $7 \cdot 4,8.5$ & 0.77 & $0.70,0.84$ & 0.73 & $0.67,0.80$ & 0.95 & $0.89,1.00$ & 82 & 0 \\
\hline $\mathrm{Ca}(\mathrm{mg})$ & 990 & 18 & 1058 & 16 & $-6 \cdot 5$ & $-7 \cdot 9,-5 \cdot 1$ & 0.42 & $0.34,0.49$ & 0.47 & $0.39,0.55$ & 0.60 & $0.48,0.71$ & 66 & 3 \\
\hline $\mathrm{Fe}(\mathrm{mg})$ & & & & & & & & & & & & & & \\
\hline Total & 11.9 & 0.2 & 11.4 & 0.2 & 4.8 & $4.7,4.9$ & 0.30 & $0.23,0.38$ & 0.34 & $0.25,0.42$ & 0.55 & $0.37,0.71$ & 66 & 4 \\
\hline Haem & $1 \cdot 2$ & 0.0 & 0.8 & 0.0 & $43 \cdot 2$ & $43 \cdot 1,43 \cdot 4$ & 0.29 & $0.22,0.35$ & 0.27 & $0.21,0.34$ & 0.51 & $0.34,0.66$ & 66 & 3 \\
\hline Non-haem & $10 . \overline{7}$ & 0.2 & 10.5 & 0.2 & $1 . \overline{9}$ & $1 \cdot 8,2 \cdot 1$ & 0.35 & $0.27,0.43$ & 0.41 & $0.32,0.50$ & 0.63 & $0.46,0.78$ & 67 & 3 \\
\hline $\mathrm{K}(\mathrm{mg})$ & 3543 & 48 & 3424 & 40 & 3.7 & $1.5,5.8$ & 0.44 & $0.37,0.52$ & 0.42 & $0.35,0.49$ & 0.59 & $0.48,0.70$ & 66 & 3 \\
\hline $\mathrm{Mg}(\mathrm{mg})$ & 378 & 5 & 368 & 5 & 3.0 & $2 \cdot 3,3 \cdot 7$ & 0.48 & $0.40,0.55$ & 0.48 & $0.40,0.56$ & 0.65 & $0.54,0.75$ & 66 & 2 \\
\hline Retinol ( $\mu \mathrm{g})$ & 511 & 18 & 531 & 22 & $-3 \cdot 2$ & $-5 \cdot 6,-0 \cdot 8$ & 0.20 & $0.13,0.26$ & 0.34 & $0.22,0.45$ & 0.41 & $0.21,0.60$ & 61 & 5 \\
\hline Vitamin $B_{1}(\mathrm{mg})$ & 1.1 & 0.0 & 1.0 & 0.0 & 6.7 & $6 \cdot 6,6 \cdot 7$ & 0.21 & $0.14,0.28$ & 0.27 & $0.17,0.36$ & 0.38 & $0.20,0.55$ & 62 & 4 \\
\hline Vitamin $B_{2}(\mathrm{mg})$ & 1.6 & 0.0 & 1.5 & 0.0 & $5 \cdot 8$ & $5.7,5.9$ & 0.34 & $0.26,0.42$ & 0.36 & $0.28,0.45$ & 0.46 & $0.33,0.58$ & 66 & 2 \\
\hline Vitamin $\mathrm{B}_{6}(\mathrm{mg})$ & 1.9 & 0.0 & 1.7 & 0.0 & 11.0 & $10 \cdot 9,11 \cdot 1$ & 0.28 & $0.20,0.36$ & 0.34 & $0.24,0.43$ & 0.39 & $0.25,0.51$ & 66 & 3 \\
\hline Vitamin $B_{12}(\mu \mathrm{g})$ & $5 \cdot 1$ & 0.1 & 4.7 & 0.2 & 9.2 & $9 \cdot 0,9.4$ & 0.28 & $0.22,0.35$ & 0.46 & $0.36,0.56$ & 0.55 & $0.36,0.73$ & 62 & 4 \\
\hline Vitamin C (mg) & 112 & 3 & 102.5 & $2 \cdot 7$ & 9.8 & $9.0,10.5$ & 0.34 & $0.27,0.41$ & 0.45 & $0.36,0.55$ & 0.63 & $0.46,0.79$ & 64 & 4 \\
\hline Vitamin D $(\mu \mathrm{g})$ & 3.8 & $2 \cdot 1$ & $3 \cdot 3$ & $0 \cdot 1$ & 14.5 & $14.4,14.7$ & 0.27 & $0.20,0.34$ & 0.38 & $0.28,0.48$ & 0.43 & $0.28,0.58$ & 62 & 3 \\
\hline Vitamin E (mg) & 13.4 & 0.3 & $12 \cdot 5$ & 0.3 & 7.4 & $7 \cdot 2,7 \cdot 6$ & 0.27 & $0.19,0.34$ & 0.34 & $0.25,0.43$ & 0.46 & $0.30,0.62$ & 61 & 5 \\
\hline Folic acid $(\mu \mathrm{g})$ & 301 & 5 & 256 & 4 & 17.8 & $17 \cdot 0,18.6$ & $0 \cdot 30$ & $0.23,0.38$ & 0.27 & $0.20,0.34$ & 0.45 & $0.31,0.58$ & 64 & 3 \\
\hline
\end{tabular}

correlation coefficient $(95 \% \mathrm{Cl})$ estimated as the correlation coefficient $/ \sqrt{ } \mathrm{ICC}_{24 \mathrm{hP}}$
col 
Table 3. Absolute intakes of food groups in the FFQ-NL 1.0 and the telephone-based 24-h recalls (24hR) and the relative difference, correlation coefficients and cross-classification between the FFQ-NL 1.0 and telephone-based $24 \mathrm{hR} \mathrm{R}^{\star}$

(Mean values with their standard errors; group-level bias, correlation coefficient, attenuation factors, de-attenuated correlation coefficient and $95 \%$ confidence intervals)

\begin{tabular}{|c|c|c|c|c|c|c|c|c|c|c|c|c|c|c|}
\hline & \multicolumn{4}{|c|}{ Absolute intake (g) } & \multirow[b]{3}{*}{$\begin{array}{l}\text { Group-level } \\
\text { bias }(\%)\end{array}$} & \multirow[b]{3}{*}{$95 \% \mathrm{Cl}$} & \multirow[b]{3}{*}{$\begin{array}{l}\text { Correlation } \\
\text { coefficient }\end{array}$} & \multirow[b]{3}{*}{$95 \% \mathrm{Cl}$} & \multirow[b]{3}{*}{$\begin{array}{l}\text { Attenuation } \\
\text { factors }\end{array}$} & \multirow[b]{3}{*}{$95 \% \mathrm{Cl}$} & \multirow[b]{3}{*}{$\begin{array}{c}\text { De-attenuated correlation } \\
\text { coefficient }\end{array}$} & \multirow[b]{3}{*}{$95 \% \mathrm{Cl}$} & \multirow{2}{*}{\multicolumn{2}{|c|}{ Cross-classification (\%) }} \\
\hline & \multicolumn{2}{|c|}{$\begin{array}{c}\text { FFQ-NL } \\
1.0\end{array}$} & \multicolumn{2}{|c|}{$24 \mathrm{hR}$} & & & & & & & & & & \\
\hline & Mean & SE & Mean & SE & & & & & & & & & $\begin{array}{l}\text { Same or } \\
\text { adjacent } Q\end{array}$ & $\begin{array}{l}\text { Extreme } \\
Q\end{array}$ \\
\hline$n$ & 383 & & 376 & & & & 37 & & 37 & & 376 & & 376 & 376 \\
\hline Potatoes & 81 & 3 & 65 & 3 & $23 \cdot 3$ & $22 \cdot 2,24 \cdot 5$ & 0.27 & $0.21,0.34$ & 0.44 & $0.34,0.54$ & 0.28 & $0.18,0.38$ & 62 & 2 \\
\hline $\begin{array}{c}\text { Non-alcoholic } \\
\text { beverages }\end{array}$ & 1723 & 31 & 1765 & 31 & $-2 \cdot 1$ & $-4 \cdot 1,0 \cdot 0$ & 0.59 & $0.51,0.67$ & 0.59 & $0.51,0.67$ & 0.60 & $0.53,0.66$ & 70 & 2 \\
\hline $\begin{array}{l}\text { Bread and bread } \\
\text { products }\end{array}$ & 133 & 3 & 138 & 3 & -3.9 & $-4 \cdot 7,-3 \cdot 1$ & 0.61 & $0.54,0.68$ & 0.58 & $0.51,0.65$ & 0.62 & $0.55,0.68$ & 78 & 2 \\
\hline Eggs & 19 & 1 & 13 & 1 & 44.7 & $43 \cdot 9,45 \cdot 5$ & 0.31 & $0.25,0.37$ & 0.47 & $0.37,0.56$ & 0.32 & $0.22,0.41$ & 65 & 2 \\
\hline Fruits & 190 & 6 & 181 & 7 & $4 \cdot 8$ & $3 \cdot 4,6 \cdot 2$ & 0.66 & $0.59,0.74$ & 0.69 & $0.62,0.77$ & 0.67 & $0.61,0.72$ & 70 & 3 \\
\hline Cake and cookies & 28 & 1 & 40 & 2 & -31.9 & $-32 \cdot 5,-31 \cdot 3$ & 0.31 & $0.24,0.38$ & 0.65 & $0.51,0.80$ & 0.33 & $0.23,0.42$ & 64 & 5 \\
\hline Cereals & 75 & 3 & 54 & 3 & 37.5 & $36 \cdot 3,38 \cdot 8$ & 0.23 & $0.16,0.30$ & 0.33 & $0.23,0.42$ & 0.24 & $0.14,0.33$ & 67 & 2 \\
\hline Vegetables & 140 & 4 & 158 & 5 & -11.1 & $-12 \cdot 1,-10 \cdot 1$ & 0.29 & $0.21,0.36$ & 0.48 & $0.36,0.60$ & 0.53 & $0.36,0.69$ & 61 & 5 \\
\hline $\begin{array}{l}\text { Savoury sandwich } \\
\text { fillings }\end{array}$ & 3 & 0 & 3 & 0 & $16 \cdot 4$ & $15 \cdot 8,16 \cdot 9$ & 0.48 & $0.40,0.56$ & 0.52 & $0.44,0.61$ & 0.49 & $0.40,0.56$ & 84 & 0 \\
\hline Cheese & 18 & 1 & 35 & 1 & -55.6 & $-55.9,-55.2$ & 0.15 & $0.08,0.22$ & 0.33 & $0.18,0.48$ & $0 \cdot 16$ & $0.05,0.26$ & 59 & 6 \\
\hline $\begin{array}{l}\text { Milk and milk } \\
\text { products }\end{array}$ & 369 & 12 & 316 & 10 & $16 \cdot 9$ & $15 \cdot 1,18 \cdot 7$ & 0.61 & $0.53,0.68$ & 0.51 & $0.44,0.57$ & 0.61 & $0.55,0.67$ & 73 & 1 \\
\hline Nuts, seeds, snacks & 23 & 1 & 23 & 1 & $-1 \cdot 4$ & $-2 \cdot 1,-0.7$ & 0.20 & $0.14,0.27$ & 0.34 & $0.23,0.44$ & 0.20 & $0.10,0.30$ & 62 & 2 \\
\hline Legumes & 22 & 2 & 6 & 1 & 274.2 & $272 \cdot 2,276 \cdot 1$ & 0.13 & $0.07,0.19$ & 0.13 & $0.07,0.20$ & $0 \cdot 13$ & $0.03,0.23$ & 62 & 0 \\
\hline Composite dishes & 11 & 1 & 39 & 3 & $-72 \cdot 0$ & $-72 \cdot 7,-71 \cdot 4$ & 0.06 & $0.00,0.12$ & 0.44 & $0.00,0.90$ & 0.06 & $0.00,0.16$ & 75 & 0 \\
\hline Soups & 61 & 4 & 62 & 4 & -0.8 & $-2 \cdot 3,0.8$ & 0.18 & $0.11,0.24$ & 0.25 & $0.16,0.35$ & 0.25 & $0.11,0.38$ & 67 & 2 \\
\hline $\begin{array}{l}\text { Soya, vegetarian } \\
\text { products }\end{array}$ & 20 & 3 & 17 & 3 & $17 \cdot 9$ & $15 \cdot 7,20 \cdot 1$ & 0.99 & $0.95,1.12$ & 0.72 & $0.66,0.77$ & 0.84 & $0.77,0.90$ & 87 & 0 \\
\hline $\begin{array}{l}\text { Sugar, honey, jams, } \\
\text { candy }\end{array}$ & 26 & 1 & 29 & 1 & $-9 \cdot 8$ & $-10 \cdot 3,-9 \cdot 2$ & 0.38 & $0.31,0.46$ & 0.54 & $0.44,0.65$ & 0.38 & $0.29,0.47$ & 71 & 4 \\
\hline Fats, oils, sauces & 40 & 1 & 39 & 1 & 3.4 & $2 \cdot 9,4 \cdot 0$ & 0.18 & $0.12,0.25$ & 0.25 & $0.16,0.34$ & 0.18 & $0.08,0.28$ & 59 & 4 \\
\hline Fish & 28 & 2 & 26 & 2 & 7.6 & $6 \cdot 6,8 \cdot 7$ & 0.27 & $0.20,0.34$ & 0.52 & $0.39,0.65$ & 0.28 & $0.18,0.37$ & 65 & 1 \\
\hline Meat & 105 & 4 & 74 & 3 & $41 \cdot 1$ & $39 \cdot 9,42 \cdot 2$ & 0.38 & $0.31,0.45$ & 0.34 & $0.28,0.40$ & 0.38 & $0.29,0.47$ & 68 & 2 \\
\hline
\end{tabular}

ICC, intraclass correlation coefficient.

* \% Group-level bias $=($ mean intake FFQ-NL 1.0/mean value $24 \mathrm{hR}) \times 100-100$; attenuation factor $(95 \% \mathrm{Cl})$ estimated as the slope in the linear regression of the reported intake from $24 \mathrm{hR}$ on the reported intake from FFQ; de-attenuated correlation coefficient $(95 \% \mathrm{Cl})$ estimated as the correlation coefficient $\sqrt{ } \mathrm{ICC}_{24 \mathrm{~h}}$. 
Table 4. Validity measures of reported intakes of protein and potassium by FFQ-NL 1.0 as compared with their urinary recovery biomarkers*

(Mean values with their standard errors; estimates and $95 \%$ confidence intervals)

\begin{tabular}{|c|c|c|c|c|}
\hline & \multicolumn{2}{|c|}{ Protein $(\mathrm{g} / \mathrm{d})$} & \multicolumn{2}{|c|}{$\mathrm{K}(\mathrm{mg} / \mathrm{d})$} \\
\hline & Mean & SE & Mean & SE \\
\hline \multirow{4}{*}{$\begin{array}{l}n \\
\text { Intake FFQ-NL } 1.0 \\
\text { Intake based on excretion }\end{array}$} & \multicolumn{2}{|c|}{362} & \multicolumn{2}{|c|}{363} \\
\hline & $82 \cdot 2$ & 1.3 & 3568 & 48 \\
\hline & $97 \cdot 7$ & 1.4 & 3747 & 58 \\
\hline & Estimate & $95 \% \mathrm{Cl}$ & Estimate & $95 \% \mathrm{Cl}$ \\
\hline$\%$ Group-level bias & $-15 \cdot 9$ & $-16 \cdot 3,-15 \cdot 5$ & $-4 \cdot 8$ & $-7 \cdot 1,-2 \cdot 4$ \\
\hline Pearson's correlation coefficient & 0.40 & $0.31,0.48$ & 0.35 & $0.26,0.44$ \\
\hline Attenuation factor & 0.46 & $0.35,0.57$ & 0.44 & $0.32,0.55$ \\
\hline Adjusted attenuation factor & 0.28 & $0.18,0.38$ & 0.39 & $0.26,0.51$ \\
\hline ICC recovery biomarker & 0.34 & $0.22,0.47$ & 0.36 & $0.24,0.50$ \\
\hline De-attenuated correlation coefficient & 0.69 & $0.53,0.83$ & 0.58 & $0.43,0.73$ \\
\hline
\end{tabular}

ICC, intraclass correlation coefficient.

* \% Group-level bias = (mean intake FFQ-NL 1.0/mean value recovery biomarker) $\times 100-100$; attenuation factor $(95 \% \mathrm{Cl}$ ) estimated as the slope in the linear regression of the biomarker on the reported intake; attenuation factor adjusted for age, sex, BMI and educational attainment (low/medium/high); de-attenuated correlation coefficient $(95 \% \mathrm{Cl})$ estimated as the correlation coefficient $/ \sqrt{ } / \mathrm{ICC}_{\text {recovery biomarker. }}$

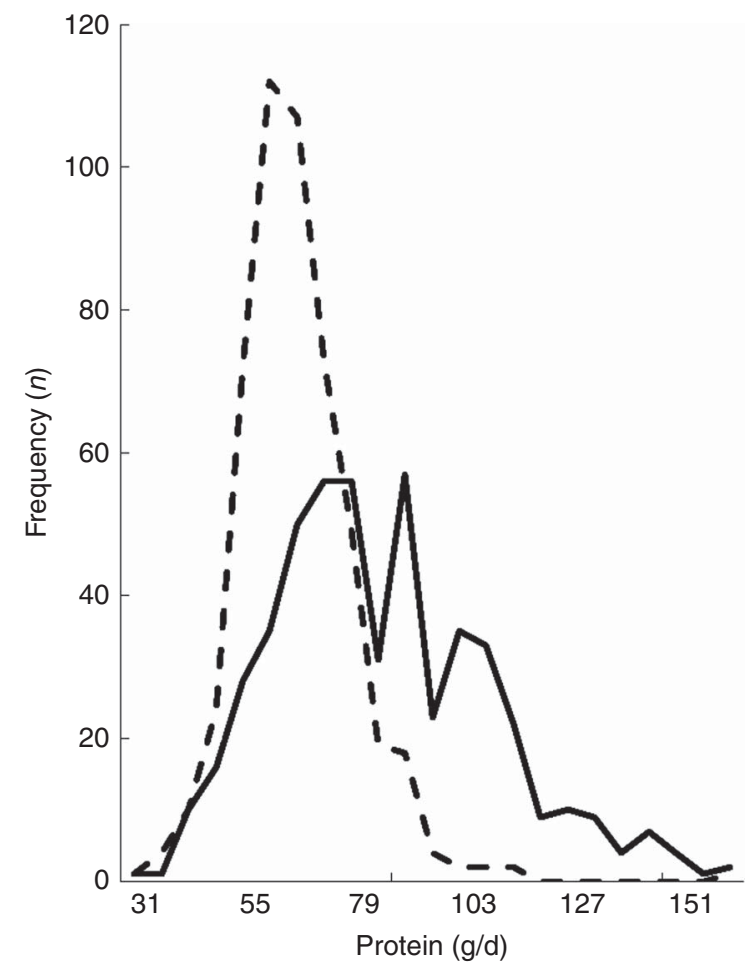

Fig. 2. Estimated distribution of protein intake from FFQ-NL $1.0(\mathrm{~g} / \mathrm{d},-\longrightarrow)$ and intake based on excretion ( $\mathrm{g} / \mathrm{d}, \cdots-\cdot-\cdot-\cdot)$.

n-3 fatty acids, correlations were higher among subjects with $\mathrm{BMI}<25 \mathrm{~kg} / \mathrm{m}^{2}$; for fatty fish, correlations were higher among men and subjects with higher educational level (data not shown).

\section{Reproducibility of the FFQ-NL 1.0}

At the group level, the replicate FFQ showed comparable intakes of most food groups (Table 6). On average, intakes

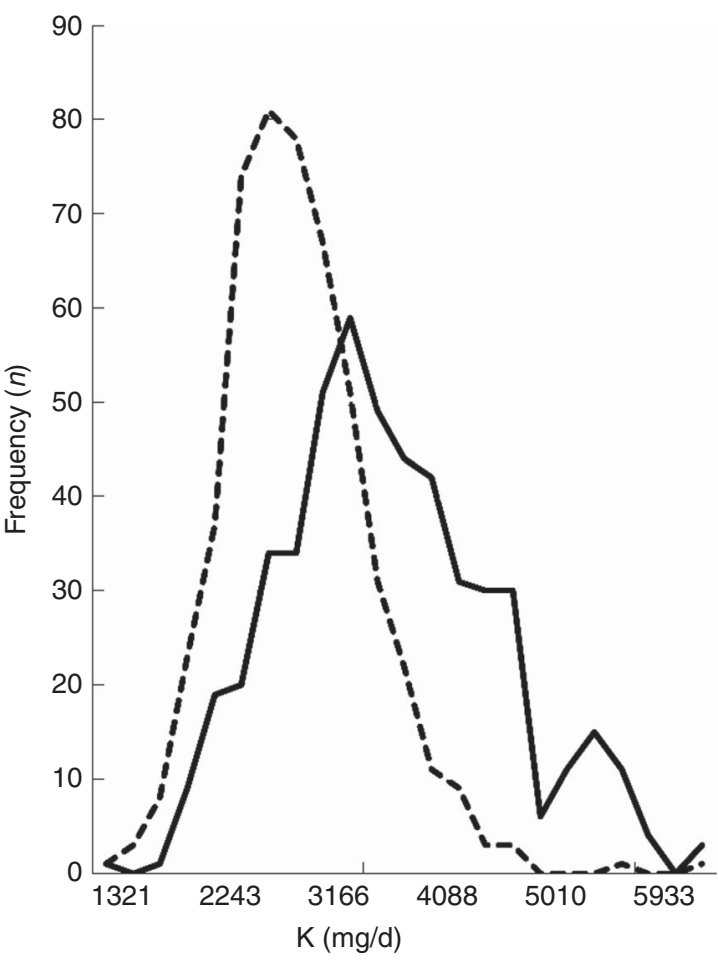

Fig. 3. Estimated distribution of potassium intake from FFQ-NL 1.0 $(\mathrm{mg} / \mathrm{d}, \longrightarrow)$ and intake based on excretion $(\mathrm{mg} / \mathrm{d}, \ldots . . . . . . .$.$) .$

of cake and cookies, savoury sandwich fillings, legumes, nuts, seeds and snacks, and fish were higher in the replicate FFQ, but intakes of soup and soya and vegetarian products were lower. Correlation coefficients showed good agreement, ranging from 0.43 for legumes to 0.85 for soya and vegetarian products. For nutrient intakes (data not shown), relative differences in intakes between the first and the second assessment of the FFQ were negligible, and correlation coefficients were high for almost all nutrients (range 0.55-0.89). 
Table 5. Pearson's correlation coefficients and cross-classification of reported intakes of (fatty) fish, fruits and vegetables in the FFQ-NL 1.0 and the related blood concentration biomarkers

(Correlation coefficients and $95 \%$ confidence intervals)

\begin{tabular}{lccccc}
\hline & $n$ & Correlation coefficient & $95 \% \mathrm{Cl}$ & Same or adjacent quintile (\%) & Extreme quintile (\%) \\
\hline Fish intake and EPA + DHA & 356 & 0.43 & $0.34,0.51$ & 67 & 2 \\
Fatty fish intake and EPA + DHA & 356 & 0.47 & $0.39,0.55$ & 69 & 2 \\
Fruit and vegetable intake and sum of carotenoids & 358 & 0.43 & $0.34,0.51$ & 69 & 3 \\
Fruit intake and sum of carotenoids & 358 & 0.37 & $0.28,0.46$ & 66 & 3 \\
Vegetable intake and sum of carotenoids & 358 & 0.29 & $0.19,0.38$ & 64 & 3 \\
Fruit and vegetable intake and $\alpha$-carotene & 358 & 0.24 & $0.14,0.34$ & 64 & 4 \\
Fruit and vegetable intake and $\beta$-carotene & 358 & 0.34 & $0.25,0.43$ & $0.32,0.49$ & 63 \\
Fruit intake and $\beta$-cryptoxanthin & 358 & 0.41 & $0.21,0.40$ & & 3 \\
Vegetable intake and lutein + zeaxanthin & 358 & 0.31 & & & 3 \\
\hline
\end{tabular}

\section{Discussion}

The present study investigated the validity of the FFQ-NL 1.0, a comprehensive, standardised, semi-quantitative FFQ for Dutch adults. Compared with the $24 \mathrm{hR}$, absolute differences were small $(<5 \%)$ for energy and macronutrients; however, the FFQ underestimated the intake of fat and overestimated the intakes of alcohol, EPA, DHA and most vitamins. For food groups, we observed only some underestimation and overestimation. Compared with their recovery markers, the FFQ underestimated protein intake by $16 \%$ and $\mathrm{K}$ by $5 \%$; the attenuation factors showed good agreement. Correlation of fruits plus vegetables and fish intakes with plasma carotenoids and $n-3$ fatty acids, respectively, was good. Overall, the validity measures were well within the range of agreement that could be expected based on the literature.

The current findings can be compared with a number of other Dutch FFQ that have been previously validated. Within 128 men and women from the Leiden Longevity Study, Streppel et $a .^{(24)}$ evaluated an FFQ against three $24 \mathrm{hR}$. Correlation coefficients varied between 0.21 and 0.78 for nutrients and for food groups between 0.00 and 0.79 . We found slightly higher correlations for macronutrients and fatty acids, but somewhat lower correlations for micronutrients. Correlations for food groups were generally higher, except for cheese, fats/oils/ savoury sauces and pastry/cake/biscuits. Goldbohm et al. validated a 150-item FFQ against three 3-d dietary records in a representative subsample of the Netherlands Cohort Study on diet and cancer. For most nutrients, correlations between 0.60 and 0.80 were observed ${ }^{(25)}$, which were generally higher compared with the present validation study. Moreover, in a reproducibility study of the FFQ, correlations were found within the range of $0 \cdot 42-0 \cdot 80^{(26)}$. Furthermore, Ocké et al. validated the FFQ used in the Dutch European Prospective Investigation into Cancer and Nutrition (EPIC) with respect to nutrients ${ }^{(27)}$ and food groups ${ }^{(28)}$ against the average of 12 monthly $24 \mathrm{hR}$. For nutrients, the median Pearson's correlation coefficient was 0.59 in men and 0.58 in women ${ }^{(27)}$; median Spearman's correlation coefficients for food groups were 0.60-0.64 for men and $0 \cdot 52-0.58$ for women ${ }^{(28)}$. De-attenuated correlation between protein intake and urinary $\mathrm{N}$ was 0.43 in men and 0.50 in women $^{(27)}$. For the FFQ-NL 1.0, we found lower correlations for both nutrients and food groups, which might be explained by the lower number of repeated $24 \mathrm{hR}-$ that is, $2 \cdot 7 /$ person on average.
Freedman et al. ${ }^{(29)}$ pooled five large US validation studies, comprising data of 2265 participants. Compared with 24-h urinary N, the FFQ under-reported protein intake by approximately 10-29\%. Furthermore, compared with 24-h K excretion, the FFQ under-reported $\mathrm{K}$ intake by $5-6 \%{ }^{(30)}$. Estimation of protein and $\mathrm{K}$ intakes in the present study was in line with the findings from these five large US studies. Attenuation factors for reported intake by FFQ were on average 0.17 for protein and $0 \cdot 25-0 \cdot 30$ for $\mathrm{K}^{(29,30)}$. Our study showed attenuation factors of 0.46 for protein and 0.44 for $\mathrm{K}$, indicating substantially lower de-attenuation of relative risks.

Plasma carotenoids are considered as biomarkers of the intake of fruits and vegetables during the previous weeks or months ${ }^{(31)}$. Al-Delaimy et al. investigated the correlation between fruit and vegetable intakes from an FFQ and plasma carotenoids within the EPIC study. The Spearman's correlation coefficient between reported fruit and vegetable intakes and total carotenoids was $0 \cdot 38^{(31)}$. Burrows et al. ${ }^{(32)}$ found a correlation between vegetable intake by FFQ and plasma $\beta$-carotene of 0.42 , between fruit intake and $\beta$-cryptoxanthin of 0.52 and a correlation of 0.30 and 0.26 between vegetable intake and lutein and zeaxanthin, respectively. For fruit and vegetable intakes by FFQ and plasma carotenoids, the present study showed correlation coefficients in the same range.

Although no accurate biomarker for total fat intake exists, EPA and DHA may serve as concentration biomarkers to evaluate fish intake ${ }^{(33)}$. Within 3009 participants from EPIC, Saadatian-Elahi et al ${ }^{(33)}$ showed a correlation coefficient of 0.29 between fatty fish intake and $n-3$ fatty acids at the individual level. In EPIC-Norfolk, Welch et al. ${ }^{(34)}$ evaluated an FFQ against $n-3$ fatty acids in blood plasma and found for reported fish intake a correlation coefficient of $0 \cdot 17$ and for fatty fish intake a correlation of 0.19 in women and 0.23 in men. With correlation coefficients of 0.43 for fish and 0.47 for fatty fish, values in the current study are higher than those found these two studies.

Dietary intake from multiple $24 \mathrm{hR}$ was assumed to best approximate true intake. However, correlated errors exist between FFQ and $24 \mathrm{hR}$, including the use of the same food composition table, and they both rely on memory and may be subject to socially desirable answers. As such, the $24 \mathrm{hR}$ may be considered an alloyed gold standard reference method. However, it is generally considered the best reference method if no recovery biomarkers are available ${ }^{(4)}$. Using $24 \mathrm{hR}$ may not remove all measurement errors of FFQ, but its use in addition 
Table 6. Reproducibility measures of food group intakes between FFQ-NL 1.0 and the replicate FFQ-NL 1.0 in 278 adults*

(Medians and percentiles 25th-75th (P25-P75); group-level bias, Pearson's correlation coefficient and $95 \%$ confidence intervals)

\begin{tabular}{|c|c|c|c|c|c|c|c|c|c|c|}
\hline & \multicolumn{4}{|c|}{ Intake $(\mathrm{g})$} & \multirow[b]{3}{*}{ Group-level bias (\%) } & \multirow[b]{3}{*}{$95 \% \mathrm{Cl}$} & \multirow[b]{3}{*}{ Pearson's correlation coefficient } & \multirow[b]{3}{*}{$95 \% \mathrm{Cl}$} & \multirow{2}{*}{\multicolumn{2}{|c|}{ Cross-classification (\%) }} \\
\hline & \multicolumn{2}{|c|}{ FFQ-NL 1.0} & \multicolumn{2}{|c|}{ Replicate FFQ } & & & & & & \\
\hline & Median & P25-P75 & Median & P25-P75 & & & & & Same or adjacent $Q$ & Extreme $Q$ \\
\hline Potatoes & 75 & $39-107$ & 70 & $36-107$ & 3.5 & $2 \cdot 4,4 \cdot 6$ & 0.70 & $0.63,0.75$ & 84 & 0 \\
\hline Non-alcoholic beverages & 1692 & $1332-2059$ & 1672 & $1305-1990$ & $1 \cdot 2$ & $-1 \cdot 0,3.5$ & 0.78 & $0.73,0.83$ & 89 & 1 \\
\hline Bread and bread products & 120 & $80-170$ & 118 & $76-167$ & 3.0 & $2 \cdot 0,4 \cdot 0$ & 0.84 & $0.80,0.87$ & 93 & 0 \\
\hline Eggs & 14 & $7-29$ & 14 & $7-29$ & -0.6 & $-1 \cdot 3,0 \cdot 1$ & 0.72 & $0.66,0.77$ & 82 & 0 \\
\hline Fruits & 206 & $102-238$ & 214 & $100-250$ & $-4 \cdot 0$ & $-5 \cdot 6,-2 \cdot 5$ & 0.76 & $0.70,0.80$ & 88 & 1 \\
\hline Cake and cookies & 23 & $13-40$ & 22 & $11-42$ & $-5 \cdot 4$ & $-6 \cdot 1,-4 \cdot 6$ & 0.63 & $0.56,0.70$ & 86 & 1 \\
\hline Cereals & 52 & 29-106 & 52 & $28-101$ & $-1 \cdot 8$ & $-3.0,-0.6$ & 0.61 & $0.53,0.68$ & 82 & 0 \\
\hline Vegetables & 138 & $90-188$ & 127 & $81-194$ & -0.8 & $-1.0,0.5$ & 0.67 & $0.59,0.73$ & 78 & 3 \\
\hline Savoury sandwich fillings & 0 & $0-4$ & 0 & $0-6$ & $-18 \cdot 2$ & $-18 \cdot 7,-17 \cdot 6$ & 0.63 & $0.55,0.69$ & 92 & 0 \\
\hline Cheese & 10 & $5-21$ & 11 & $5-21$ & $9 \cdot 4$ & $8 \cdot 7,10 \cdot 1$ & 0.55 & $0.46,0.63$ & 75 & 1 \\
\hline Milk and milk products & 344 & $200-511$ & 328 & 194-482 & 3.9 & $1.9,5.9$ & 0.75 & $0.70,0.80$ & 89 & 0 \\
\hline Nuts, seeds and snacks & 16 & $7-33$ & 17 & $6-35$ & $-7 \cdot 3$ & $-8 \cdot 0,-6 \cdot 6$ & 0.64 & $0.56,0.70$ & 85 & 1 \\
\hline Legumes & 16 & $4-22$ & 16 & $4-26$ & $-13 \cdot 0$ & $-14 \cdot 0,-12 \cdot 0$ & 0.43 & $0.33,0.52$ & 82 & 2 \\
\hline Composite dishes & 5 & $0-19$ & 3 & $0-19$ & 0.3 & $-0.5,1.0$ & 0.71 & $0.46,0.76$ & 87 & 0 \\
\hline Soups & 30 & $8-48$ & 29 & $10-48$ & $5 \cdot 8$ & $4 \cdot 1,7.5$ & 0.76 & $0.71,0.81$ & 84 & 1 \\
\hline Soya and vegetarian products & 0 & $0-11$ & 0 & $0-8$ & 8.4 & $6 \cdot 4,10 \cdot 4$ & 0.85 & $0.81,0.88$ & 89 & 0 \\
\hline Sugar, honey, jams, candy & 21 & $9-37$ & 23 & $12-36$ & $-4 \cdot 1$ & $-4 \cdot 7,-3.4$ & 0.69 & $0.62,0.74$ & 84 & 1 \\
\hline Fats, oils and sauces & 34 & $22-53$ & 33 & $21-52$ & $1 \cdot 1$ & $0.4,1.7$ & 0.60 & $0.51,0.67$ & 75 & 1 \\
\hline Fish & 18 & $9-34$ & 18 & $9-43$ & $-6 \cdot 7$ & $-7 \cdot 6,-5 \cdot 7$ & 0.71 & $0.65,0.77$ & 88 & 0 \\
\hline Meat & 93 & $47-137$ & 85 & $50-137$ & -0.6 & $-1 \cdot 8,0 \cdot 6$ & 0.76 & $0.70,0.80$ & 86 & 0 \\
\hline
\end{tabular}

*\% Group-level bias $=($ mean intake FFQ-NL 1.0/mean intake replicate FFQ-NL 1.0) $\times 100-100$ 
to an FFQ will improve diet-disease associations ${ }^{(4,35)}$. Furthermore, the use of multiple $24 \mathrm{hR}$ is recommended above a single recall $^{(29,30)}$. Compared with the $24 \mathrm{hR}$, the FFQ-NL 1.0 seemed to under-report the intakes of fat and high-fat foods, particularly SFA, trans-fatty acids, cake and cookies, and cheese. The current study population comprised more women and highly educated persons than the general population. As a result, total variation in fat intake might be lower, corresponding with lower validity measures. Furthermore, subjects over-reported their intakes of legumes, soya and vegetarian products, alcohol and most micronutrients in the FFQ-NL 1.0 compared with the $24 \mathrm{hR}$. Subjects also tended to under-report the intake of composite dishes, which may be due to coding differences. In the FFQ-NL 1.0, the covered variance in intake was $<80 \%$ for EPA, DHA, trans-fatty acids and vitamins $B_{1}, B_{2}$, $\mathrm{B}_{6}, \mathrm{C}, \mathrm{D}, \mathrm{E}$ and folate equivalents, which is likely reflected in the lower validity measures found for these nutrients. To increase the covered variance of particularly $\mathrm{B}$ vitamins, a new version of the FFQ-NL 1.0 is now being developed including a few additional food items. Hence, the validity of the improved FFQ is expected to be similar or higher.

Age and BMI may contribute to intake-related bias in the FFQ as well as correlated errors between FFQ and $24 \mathrm{hR}^{(35)}$. Freedman et al. observed that a higher BMI was associated with a larger degree of under-reporting; having a lower educational level was also associated with more under-reporting. Furthermore, age above 59 years was associated with less underreporting ${ }^{(29,30)}$. Indeed, the current study showed that attenuation factors were generally higher among older subjects and subjects with normal BMI, as well as among men and subjects with higher education.

The NQplus study population comprises a sample of highly educated and committed participants, who have become familiar with dietary assessment methods and with blood and urine collections. Hence, the participants may have been more diligent and accurate in recording their intakes and collecting their urine samples than the general population. However, the study benefitted from the multiple reference methods to which the FFQ could be validated: urinary recovery biomarkers, blood concentration markers and multiple $24 \mathrm{hR}$, as well as the advanced statistical methods, which were used to assess validity.

To provide a reliable and correct measure of dietary intake in free-living populations is the largest challenge of nutritional epidemiology. Measurement errors in FFQ cannot be prevented, but they can be quantified and accounted for. Therefore, it is always recommended to use objective biological markers of dietary intake or $24 \mathrm{hR}$ complementary to an FFQ. Furthermore, an external validation study such as the present one is also essential in identifying and quantifying measurement errors and how diet-disease associations are affected. Attenuation directly affects the observed relative risk as well as the necessary sample size to detect these diet-disease relationships ${ }^{(36)}$. Thus, future epidemiological studies using the FFQ-NL 1.0 can apply the attenuation factors presented here to calculate the sample size needed for desired statistical power and to adjust for observed relative risks.

Validity coefficients or de-attenuated correlation coefficients may also be used to quantify the impact of measurement error on diet-disease relationships ${ }^{(35)}$, and were estimated as the correlation coefficients between the FFQ-NL 1.0 and the reference instrument divided by the square root of the ICC of replicates of the reference method. It is assumed that if the time between replicate urine collections and the $24 \mathrm{hR}$ is not too close, the errors in replicates are independent and they provide a measure of within-person variation within the biomarker ${ }^{(37)}$. However, within-person variation of the $24 \mathrm{hR}$, characterised by a low ICC, was high, which may have led to an overestimation of the de-attenuated correlation coefficient. This may be because of the correlated errors between FFQ and $24 \mathrm{hR}$

In conclusion, the overall validity of the newly developed FFQ-NL 1.0 was acceptable to good, and the FFQ was able to adequately rank subjects according to their dietary intake. Therefore, the FFQ-NL 1.0 is well suited for future use within Dutch cohort studies among adults. As a future application, the FFQ-NL 1.0 can be used to improve the pooling of results from individual studies using the FFQ.

\section{Acknowledgements}

This study was financially supported by the Dutch Biobanking and Biomolecular Research Infrastructure project (BBMRI-NL), a Research Infrastructure financed by the Dutch Government (NWO 184.021.007).

M. C. O., E. J. M. F. and P. C. D. designed the study. A. G., J. H. M. d. V. and E. J. M. F. acquired the data. D. S. carried out the analysis, interpreted the data and drafted the article. All the authors interpreted the data, critically reviewed the article for important intellectual content and gave final approval of the version to be published.

All authors declare no conflicts of interest.

\section{Supplementary material}

For supplementary material/s referred to in this article, please visit http://dx.doi.org/doi:10.1017/S0007114516002749

\section{References}

1. Satija A, Yu E, Willett WC, et al. (2015) Understanding nutritional epidemiology and its role in policy. Adv Nutr $\mathbf{6}$, $5-18$.

2. Willett WC (1998) Chapter 6. Reproducibility and validity of food-frequency questionnaires. In Nutritional Epidemiology, 2nd ed. pp. 321-346 [W Willett, editor]. New York: Oxford University Press.

3. Schatzkin A, Subar AF, Moore S, et al. (2009) Observational epidemiologic studies of nutrition and cancer: the next generation (with better observation). Cancer Epidemiol Biomarkers Prev 18, 1026-1032.

4. Freedman LS, Schatzkin A, Midthune D, et al. (2011) Dealing with dietary measurement error in nutritional cohort studies. J Natl Cancer Inst 103, 1086-1092.

5. Cade J, Thompson R, Burley V, et al. (2002) Development, validation and utilisation of food-frequency questionnaires a review. Public Health Nutr 5, 567-587. 
6. van Lee L, Feskens EJ, Meijboom S, et al. (2016) Evaluation of a screener to assess diet quality in the Netherlands. Br J Nutr 115, 517-526.

7. van Rossum CTM, Fransen HP, Verkaik-Kloosterman J, et al. (2011) Dutch National Food Consumption Survey 2007-2010: Diet of Children and Adults 7 to 69 years. Bilthoven, the Netherlands: RIVM

8. NEVO-tabel (2011) Nederlands Voedingsstoffen-tabel (NEVOtabel) 2011 (Dutch Food Composition Table 2011) version 3. Bilthoven, the Netherlands: RIVM/Dutch Nutrition Centre.

9. Willett WC (2013) Chapter 13: Issues in analysis and interpretation of dietary data. In Nutritional Epidemiology, 3rd ed., pp. 306 [W Willett, editor]. New York: Oxford University Press.

10. Conway JM, Ingwersen LA \& Moshfegh AJ (2004) Accuracy of dietary recall using the USDA five-step multiple-pass method in men: an observational validation study. J Am Diet Assoc 104, 595-603.

11. Conway JM, Ingwersen LA, Vinyard BT, et al. (2003) Effectiveness of the US Department of Agriculture 5-step multiplepass method in assessing food intake in obese and nonobese women. Am J Clin Nutr 77, 1171-1178.

12. Moshfegh AJ, Rhodes DG, Baer DJ, et al. (2008) The US Department of Agriculture Automated Multiple-Pass Method reduces bias in the collection of energy intakes. Am J Clin Nutr 88, 324-332.

13. NEVO-online versie 2011/3.0 R, Bilthoven. http://www.rivm.nl/ Onderwerpen/N/Nederlands_Voedingsstoffenbestand/Contact/ Voorwaarden_NEVO_Online_versie_2011_3_0

14. Jakobsen J, Ovesen L, Fagt S, et al. (1997) Para-aminobenzoic acid used as a marker for completeness of 24 hour urine: assessment of control limits for a specific HPLC method. Eur J Clin Nutr 51, 514-519.

15. Subar AF, Midthune D, Tasevska N, et al. (2013) Checking for completeness of 24-h urine collection using para-amino benzoic acid not necessary in the Observing Protein and Energy Nutrition study. Eur J Clin Nutr 67, 863-867.

16. Bingham SA (2003) Urine nitrogen as a biomarker for the validation of dietary protein intake. J Nutr 133, Suppl. 3, 921S-924S.

17. Freisling H, van Bakel MM, Biessy C, et al. (2012) Dietary reporting errors on $24 \mathrm{~h}$ recalls and dietary questionnaires are associated with BMI across six European countries as evaluated with recovery biomarkers for protein and potassium intake. Br J Nutr 107, 910-920.

18. Leenders M, Leufkens AM, Siersema PD, et al. (2014) Plasma and dietary carotenoids and vitamins $\mathrm{A}, \mathrm{C}$ and $\mathrm{E}$ and risk of colon and rectal cancer in the European Prospective Investigation into Cancer and Nutrition. Int $J$ Cancer 135, 2930-2939.

19. Lu Y, Vaarhorst A, Merry AH, et al. (2012) Markers of endogenous desaturase activity and risk of coronary heart disease in the CAREMA cohort study. PLOS ONE 7, e41681.

20. Jenab M, Slimani N, Bictash M, et al. (2009) Biomarkers in nutritional epidemiology: applications, needs and new horizons. Hum Genet 125, 507-525.

21. Hankinson SE, Manson JE, Spiegelman D, et al. (1995) Reproducibility of plasma hormone levels in postmenopausal women over a 2-3-year period. Cancer Epidemiol Biomarker Prev 4, 649-654.

22. Kromrey JD, Fay RH \& Bellara AP (2008) Paper PO-089: ATTENUATE: a SAS macro for computing confidence intervals for disattenuated correlation coefficients. SESUG Proceedings,
SESUC, Inc. http://analytics.ncsu.edu/sesug/2008/PO-089.pdf (accessed July 2015).

23. Willett WC (2013) Nutritional Epidemiology, 3rd ed. New York: Oxford University Press.

24. Streppel MT, de Vries JH, Meijboom S, et al. (2013) Relative validity of the food frequency questionnaire used to assess dietary intake in the Leiden Longevity Study. Nutr J 12, 75 .

25. Goldbohm RA, van den Brandt PA, Brants HA, et al. (1994) Validation of a dietary questionnaire used in a large-scale prospective cohort study on diet and cancer. Eur J Clin Nutr 48, 253-265.

26. Goldbohm RA, van 't Veer P, van den Brandt PA, et al. (1995) Reproducibility of a food frequency questionnaire and stability of dietary habits determined from five annually repeated measurements. Eur J Clin Nutr 49, 420-429.

27. Ocke MC, Bueno-de-Mesquita HB, Pols MA, et al. (1997) The Dutch EPIC food frequency questionnaire. II. Relative validity and reproducibility for nutrients. Int J Epidemiol 26, Suppl. 1, S49-S58.

28. Ocke MC, Bueno-de-Mesquita HB, Goddijn HE, et al. (1997) The Dutch EPIC food frequency questionnaire. I. Description of the questionnaire, and relative validity and reproducibility for food groups. Int J Epidemiol 26, Suppl. 1, S37-S48.

29. Freedman LS, Commins JM, Moler JE, et al. (2014) Pooled results from 5 validation studies of dietary self-report instruments using recovery biomarkers for energy and protein intake. Am J Epidemiol 180, 172-188.

30. Freedman LS, Commins JM, Moler JE, et al. (2015) Pooled results from 5 validation studies of dietary self-report instruments using recovery biomarkers for potassium and sodium intake. Am J Epidemiol 181, 473-487.

31. Al-Delaimy WK, Ferrari P, Slimani N, et al. (2005) Plasma carotenoids as biomarkers of intake of fruits and vegetables: individual-level correlations in the European Prospective Investigation into Cancer and Nutrition (EPIC). Eur J Clin Nutr 59, 1387-1396.

32. Burrows TL, Hutchesson MJ, Rollo ME, et al. (2015) Fruit and vegetable intake assessed by food frequency questionnaire and plasma carotenoids: a validation study in adults. Nutrients 7, 3240-3251.

33. Saadatian-Elahi M, Slimani N, Chajes V, et al. (2009) Plasma phospholipid fatty acid profiles and their association with food intakes: results from a cross-sectional study within the European Prospective Investigation into Cancer and Nutrition. Am J Clin Nutr 89, 331-346.

34. Welch AA, Bingham SA, Ive J, et al. (2006) Dietary fish intake and plasma phospholipid $n-3$ polyunsaturated fatty acid concentrations in men and women in the European Prospective Investigation into Cancer-Norfolk United Kingdom cohort. Am J Clin Nutr 84, 1330-1339.

35. Geelen A, Souverein OW, Busstra MC, et al. (2015) Comparison of approaches to correct intake-health associations for FFQ measurement error using a duplicate recovery biomarker and a duplicate $24 \mathrm{~h}$ dietary recall as reference method. Public Health Nutr 18, 226-233.

36. Kipnis V, Subar AF, Midthune D, et al. (2003) Structure of dietary measurement error: results of the OPEN biomarker study. Am J Epidemiol 158, 14-21; discussion 22-16.

37. Preis SR, Spiegelman D, Zhao BB, et al. (2011) Application of a repeat-measure biomarker measurement error model to 2 validation studies: examination of the effect of within-person variation in biomarker measurements. Am J Epidemiol 173, 683-694. 